expression of immune checkpoints strongly associated with prognosis in many cancers. Although, immune checkpoints also have been actively studied in many hematologic malignancies, the prognostic implications of these checkpoints for hematologic malignancies remain unclear. $\mathrm{V}$-set $\mathrm{Ig}$ domain-containing 4 (VSIG4) is a newly identified B7-related family member that induces tolerance in T cells and NKT cells.

Aims: In this study, we explored the clinical significance of cancer cell-associated VSIG4 expression in MM using immunohistochemistry.

Methods: We evaluated the expression of VSIG4 in 67 extramedullary biopsies from MM patients between 2000 and 2011 at Asan Medical Center and correlated VSIG4 expression with clinical outcomes.

Results: VSIG4 expression was associated with older age and no autologous stem cell transplantation treatment $(p=0.021$ and 0.013 , respectively). The high VSIG4 expression group had a significantly worse overall survival than the low expression group $(p=0.025)$. After multivariable adjustment, higher International Staging System and VSIG4 expression were associated with a worse overall survival ( $p=0.007$ and 0.039 , respectively). Conclusion: These results suggest that VSIG4 can serve as an independent prognostic marker and can be a candidate molecule to develop targeting molecule for treatment of MM.

\section{A RETROSPECTIVE STUDY TO CLASSIFY BREAST CARCINOMAS ACCORDING TO MOLECULAR CLASSIFICATION AND TO CORRELATE THE ER, PR, HER2 RESULTS WITH THE NOTTINGHAM GRADE AND THE AXILLARY LYMPH NODE STATUS AND TO COMPARE THE NOTTINGHAM GRADE WITH AXILLARY LYMPH NODE STATUS IN BREAST CARCINOMAS}

Indika Liyanage, Leonardo D. Santos, Jim Yong Sydney South West Pathology Services (SSWPS), Liverpool, NSW, Australia

Background: Breast carcinoma (BC) is a heterogeneous disease. Gene expression analyses identify several subtypes which are of prognostic and predictive importance.

Aims: (1) to determine the prevalence of BC subtypes from SSWPS area patients according to molecular classification using immunohistochemistry (IHC) as surrogate markers and (2) to determine the relationship between certain pathological characteristics.

Methods: We reviewed 312 cases of BC diagnosed in 2013 in our department. Molecular classification of $\mathrm{BC}$ was based on Estrogen Receptor (ER), Progesterone Receptor (PR), HER2/neu (HER2), HER2 CISH and Ki-67 results. Triple negative cases were subdivided into Basal-like and Unclassified groups using CK5/6, CK14 and EGFR. The ER, PR and HER2 results were correlated with Nottingham grade (NG) and axillary lymph node (ALN) status.

Results: The commonest molecular subtype was Luminal A subtype (45\%) followed by Luminal B (38\%) [Luminal B HER2 negative $(32 \%)$ and Luminal B HER2 positive (6\%)], HER2 positive (7\%), Basal-like (9\%) and Unclassified subtype (2\%). Positive ER and PR findings were inversely correlated with higher NG $(p \leq 0.0001)$ whereas high NG was positively correlated with positive HER2 status $(p \leq 0.0001)$. There was no correlation between ALN status and ER, PR and HER2 results. A statistically significant correlation was found between higher NG and number of ALN metastasis. Majority of Luminal A subtype BCs were either of NG 1 or 2 (135 cases, 96.4\%). Amongst the NG 3 cases, majority of the BCs belonged to Luminal B HER2 negative subtype $(44.14 \%)$.

Conclusion: This study shows that molecular classification of $\mathrm{BC}$ by IHC is practical and is necessary for therapeutic decision and prognosis as molecular testing is not always feasible in routine laboratories.

\section{BILATERAL ADRENAL MASSES IN A DIABETIC PATIENT: AN UNUSUAL PRESENTATION OF HISTOPLASMOSIS}

Nassim Saremi ${ }^{1}$, Fereshteh Ameli ${ }^{2}$,

Wan Faiziah Wan Abdul Rahman ${ }^{3,4}$, Alfred K-Y. Lam ${ }^{1,4}$

${ }^{1}$ Cancer Molecular Pathology and Griffith Health Institute, Griffith University, Gold Coast, Australia; ${ }^{2}$ University Kebangsaan Malaysia Medical Centre, Kuala Lumpur, ${ }^{3}$ School of Medical sciences, Universiti Sains Malaysia, Kelantan, Malaysia; and ${ }^{4}$ Pathology Queensland, Gold Coast University Hospital, Gold Coast, Australia

Aims and background: Histoplasmosis is an infection disease caused by the fungus Histoplasma capsulatum and endemic in many regions such as Asia. Most infections are not clinically recognized, but rather identified as incidental radiographic or pathological findings. The aim is to describe an unusual presentation of Histoplasmosis diagnosed by adrenal biopsy.

Methods: The patient was a 73-year-old man, who has been suffering from diabetes mellitus over the last 20 years. He presented in one of rural hospital in Malaysia with fever, chills, night sweat and loss of weight. Laboratory tests for tuberculosis were negative. However, computed tomographic scan showed bilateral adrenal masses. The adrenal biopsy showed only necrotic tissue and pleomorphic nuclei. After two years of defaulted followed up, he presented again to a tertiary hospital with generalised pain and loss of weight over a two months and complained of alternative cough with mild sputum. Excisional biopsy of the adrenal mass was carried out and revealed fungal infection with morphology compatible with histoplasmosis. He was treated with Amphotericin B liposomal and showed a dramatic symptomatic improvement. Conclusion: Histoplasmosis may mimics common diseases and its presentation as bilateral adrenal masses are unusual. A high index of suspicion is needed for the correct diagnosis of the disease and in particular in endemic region.

\section{A GEOGRAPHICAL COMPARISON OF P16 EXPRESSION IN SQUAMOUS CELL CARCINOMA (SCC) OF THE TONGUE}

Laveniya Satgunaseelan ${ }^{1}$, Sohaib Virk ${ }^{2}$, Trina Lum ${ }^{1}$, N. Gopalakrishna Iyer ${ }^{4}$, Ruta Gupta ${ }^{1,3}$, Jonathan Clark ${ }^{2,3}$ ${ }^{1}$ Department of Tissue Pathology and Diagnostic Oncology, Royal Prince Alfred Hospital, ${ }^{2}$ Sydney Head and Neck Cancer Institute, Chris O'Brien Lifehouse, ${ }^{3}$ Central Clinical School, University of Sydney, Sydney, Australia; and ${ }^{4}$ National Cancer Centre, Singapore

Background: p16 expression is poorly characterised in oral SCC, unlike in oropharyngeal carcinoma, where there is known geographical variation in incidence. 